\title{
Indicadores demográficos de perros y gatos con dueño en el distrito de San Borja, Lima-Perú, 2017
}

\author{
Demographic indicators and to estimate dog and cat population in San Borja district, Lima, Peru.
}

Raquel Arellano ${ }^{1,2}$, Milagro Osorio ${ }^{3}$, María del Carmen Napurí ${ }^{3}$, Daphne León ${ }^{1,2}$, Néstor Falcón ${ }^{1,2}$

\section{RESUMEN}

El objetivo del estudio fue cuantificar los indicadores demográficos y estimar la población de perros y gatos en el distrito de San Borja. Para esto, se diseñó un estudio observacional a base de encuestas que consideró como variables: tipo de viviendas, número de personas por vivienda, tenencia y número de perros y gatos, características demográficas (sexo, edad y raza), datos reproductivos y edad de fallecimiento del último perro o gato. La recolección de la información se hizo en viviendas, que se seleccionaron con un muestreo estratificado aleatorio de manzanas o conjuntos habitacionales, encuestándose 10 viviendas en cada caso. Se recolectaron 871 encuestas, las que provinieron principalmente de casas $(57,7 \%)$ y departamentos $(40,8 \%)$. Del total de las viviendas el 54,2\% tenía perros y $11,6 \%$ gatos. El promedio de animales por vivienda fue de 1,4 para los perros y 1,7 para los gatos, entre las viviendas que los poseían. Se obtuvo una relación persona: perro de 5:1 y persona: gato de 19,5:1. La población estimada de perros fue de 22543 y de gatos de 5781 para el año 2017. El porcentaje de supervivencia de las crías estimado fue de $88,6 \pm 4,9 \%$ en perros y $88,4 \pm 9,4 \%$ en gatos y la esperanza de vida estimada fue de $9,44 \pm 1,2$ y $7,38 \pm 3,4$ años, para perros y gatos respectivamente. El principal uso de los perros $(97,9 \%)$ y gatos $(100 \%)$ fue como mascota. En perros predominó la tenencia de machos $(57,2 \%)$, de raza pura $(67,5 \%)$, no esterilizados $(70,4 \%)$ y tamaño pequeño $(45,6 \%)$. La edad promedio fue de 4,26 años. En gatos, predominaron las hembras $(55,3 \%)$, esterilizados $(63,5 \%)$ y raza cruzada $(79,6 \%)$. La edad promedio fue de 2,52 años. Estos valores aportan información demográfica de perros y gatos que servirán para mejorar la planificación de programas de vacunación antirrábica y tenencia responsable de estas poblaciones.

PALABRAS CLAVE: Gato, perro, rabia, zoonosis.

\section{SUMMARY}

The aim was to quantify demographic indicators and to estimate dog and cat population in San Borja district, Lima, Peru. For that, an observational study was designed. Information was obtained through a survey which includes variables as type of housing, number of residents per house, ownership and number of dogs and cats, animal demographic characteristics (sex, age, and breed), animal reproductive data and age of death of their last animal. To select the surveyed houses a random stratified sampling was performed between blocks of housing, 10 houses were surveyed on each block. In total, 871 surveys were collected, and were mainly from houses (57.7\%) and apartment (40.8\%). Overall $54,2 \%$ of households had dogs and $11,6 \%$ cats. The average number of dogs per house was 1.4 and was 1.7 for cats. The human: dog ratio was 5:1, and the human: cat ratio was 19.5:1. The estimated population for dogs was 22543 and for cats 5781 for 2017. The survival rate of the offspring was $88.6 \pm 4.9 \%$ for dogs and 88.4

${ }^{1}$ Grupo SAPUVET-PERÚ, Facultad de Medicina Veterinaria y Zootecnia, Universidad Peruana Cayetano Heredia. Lima, Perú.

${ }^{2}$ Laboratorio de Epidemiología y Salud Pública, Facultad de Medicina Veterinaria y Zootecnia, Universidad Peruana Cayetano Heredia. Lima, Perú.

${ }^{3}$ Municipalidad Distrital de San Borja. Lima, Perú. 
$\pm 9.4 \%$ for cats. The life expectancy was of $9.44 \pm 1.2$ for dogs and $7.38 \pm 3.4$ years for cats. The most common use for dogs $(97.9 \%)$ and cats $(100 \%)$ was as pets. Among dogs, the majority was male (57.2\%), purebred (67.5\%), non-sterilized (70.4\%), and small size (45.6\%). The average age was of 4.26 years. Among cats, the majority was females $(55.3 \%)$, sterilized (63.5\%), and crossbred (79.6\%). The average age was 2.52 years. The results provide demographic information about dogs and cats from San Borja district. The information can be useful to improve rabies vaccination planning and responsible pet ownership programs.

KEYWORDS: Cats, dogs, rabies, zoonoses.

\section{INTRODUCCIÓN}

La tenencia no responsable de los animales de compañía ocasiona un aumento no controlado en su población, lo que puede generar problemas en la salud pública (Hugues et al., 2013; León, Soriano, Arauco, y Falcón, 2014; Cuch, 2004), entre las que se encuentran la transmisión de zoonosis, la contaminación ambiental por excretas y los accidentes por mordedura (León et al., 2014).

Por ello, se consideran altamente necesarias las prácticas de programas de prevención de enfermedades y tenencia responsable de estos animales (Hugues et al., 2013; Gómez, Atehortua, y Orozco, 2007; Botero, 2009); y para intervenir en estas poblaciones es necesario conocer información demográfica, la cual puede obtenerse a base de estimaciones. Estos datos permiten efectuar el cálculo de los recursos necesarios para poder ejecutar diversos programas de tenencia responsable y prevención de enfermedades zoonóticas (León et al., 2014, Ministerio de Salud [MINSA], 2017).

En la década de los ' 70 ' se llevó a cabo el primer estudio de estimación de la población de perros en Lima, calculándose el índice persona: perro de 10,3:1 (Málaga, 1973.). Estudios recientes han aportado información por distritos. Estudios a base de encuestas en escolares realizados en San Martin de Porres y Comas encontraron una relación persona: perro de 7:1 (Arauco, Falcón, León, y Urbina, 2014) y 5,74:1 (Soriano, Nuñez, León, y Falcón, 2017) respectivamente. Otro estudio que incluye la población de perros y gatos en el distrito de Ventanilla, Callao encontró una relación persona: perro de 3,98:1 y persona: gato de 5,34:1 (Rendón et al., 2018).

A nivel de Latinoamérica se han desarrollado algunos estudios que consideran a la población de perros y gatos, como el realizado en Santa Cruz de la Sierra Bolivia donde se encontró una relación persona: perro de 4,3:1 y persona: gato de 12,2:1. (Loza, 2014). En la comuna de Lo Prado- Chile se ha encontrado una relación persona: perro de 6,8:1 y persona: gato de 9,35:1 durante el periodo 2004-2013 (Venegas, 2014). Asimismo, en la comuna de Santiago de la Región Metropolitana - Chile se halló una relación persona: perro de 7,3:1 y persona: gato de 7,4:1 (Bustamante, 2008). En la ciudad de Torreón en Coahuila - México se reportó una relación persona: perro de 3,76:1 y una relación persona: gato de 18,8:1 (Bautista, 2013).

La dinámica de la población de perros y gatos varía de acuerdo con el lugar geográfico en el que se desarrolla la estimación. Su conocimiento es importante para el desarrollo, promoción y planificación adecuada de los programas de salud preventiva, educación sanitaria y tenencia responsable de animales de compañía, los que se encuentran bajo la responsabilidad de las Municipalidades conforme señala la Ley $\mathrm{N}^{\circ} 27596$, Ley que regula el Régimen Jurídico de Canes, y su Reglamento el Decreto Supremo $\mathrm{N}^{\circ} 006-2002-\mathrm{SA}$ (Congreso de la República del Perú, 2001). En ese contexto, la Municipalidad de San Borja requiere conocer esta información por lo que el objetivo del estudio fue cuantificar los indicadores demográficos y estimar la población de perros y gatos en viviendas ubicadas en dicho distrito.

\section{MATERIAL Y MÉTODOS}

El estudio, en la fase de encuestas se realizó el mes de julio del año 2017 en el distrito de San Borja, Lima - Perú. El desarrollo de la base de datos y el análisis estadístico correspondiente fueron realizados en la Facultad de Medicina Veterinaria y Zootecnia de la Universidad Peruana Cayetano Heredia (FAVEZ$\mathrm{UPCH})$. El estudio correspondió a una investigación de tipo observacional, transversal y descriptiva.

La población de estudio estuvo constituida por las viviendas pertenecientes a 12 sectores previamente seleccionados. El tamaño de muestra se determinó mediante la fórmula de comprobación de una proporción para poblaciones desconocidas, tomando en cuenta las siguientes restricciones: nivel 
de confianza del $99 \%$, proporción referencial para las viviendas con perros y/o gatos del 50\% (valor utilizado cuando se desconoce información previa) y error máximo admisible de 5\%. El mínimo tamaño de muestra calculado fue de 664 viviendas.

El instrumento fue desarrollado, en su primera versión, por profesionales del Área de Epidemiología y Salud Pública de la FAVEZ - UPCH y la Municipalidad del distrito de San Borja siendo posteriormente validada por los profesionales en Salud Pública que laboran en las instituciones mencionadas.

Las variables que se tomaron en cuenta en el estudio fueron las siguientes:

- Tipo de viviendas (casa, departamento, otros).

- Número de personas por vivienda.

- Tenencia de perros y gatos (si o no)

- Número de perros y gatos en el hogar y características demográficas (sexo, edad y raza)

- Datos reproductivos (numero de partos, numero de crías nacidas y sobrevivientes).

- Control de reproducción (esterilizado o no esterilizado)

- Edad de fallecimiento de un último animal de compañía, si lo hubieran tenido.

Para el proceso de encuestado se contó con el apoyo del personal de la Municipalidad de San Borja y de estudiantes del voluntariado SAPUVET-PERÚ de la FAVEZ-UPCH. En cada manzana seleccionada se eligió un mínimo de 10 viviendas siguiendo los criterios de un muestreo estratificado aleatorio. En el caso en que la manzana correspondiera a un conjunto habitacional, se seleccionó los departamentos ubicados en el primer y segundo piso de dicha edificación. Cuando una manzana correspondió mayoritariamente a una estructura no habitada o a un centro comercial, esta se descartó seleccionándose una manzana contigua.

Los encuestadores fueron capacitados en el llenado de la encuesta y la selección de las viviendas a encuestar. Además del material de encuesta se proporcionó un plano, en el que se indicaban las manzanas seleccionadas para el desarrollo de la encuesta y un distintivo que los acreditaba como personal de la Municipalidad.

La información recogida en las encuestas fue transferida a una base de datos en el programa Microsoft Excel y analizada en el programa estadístico SPSS 22.0. Se calculó la proporción de viviendas del distrito que poseían animales de compañía, el promedio de ellos por vivienda y la relación persona: perro y persona: gato. Las relaciones persona: perro y persona: gato sirven para estimar la población de perros y gatos que habitarían en San Borja. Para ello se utilizó la proyección de población humana del Instituto Nacional de Estadística e Informática (Instituto Nacional de Estadística e Informática [INEI], 2017).

Los resultados de las variables cualitativas (vivienda, sexo, raza, control reproductivo, utilidad) fueron resumidos mediante tablas de frecuencia y las variables cuantitativas (edad, número de crías al parto, esperanza de vida de perros y gatos) se resumieron mediante estadística descriptiva empleando la media aritmética, desviación estándar y valores extremos de la variable en estudio.

\section{RESULTADOS}

El estudio de investigación logró recolectar 871 encuestas válidas de un total de 1287 efectuadas en viviendas seleccionadas en el distrito. El tipo de viviendas a las que se encuestó fueron casas $(57,7 \%)$, seguido de departamentos o complejos habitacionales $(40,8 \%)$ y finalmente otros tipos de vivienda que no se clasificó como casas ni departamentos $(1,5 \%)$. El $54,2 \%(472 / 871)$ y $11,6 \%(101 / 871)$ de los encuestados mencionaron tener perros y gatos en sus viviendas (tabla 1).

El porcentaje de viviendas con uno y dos perros fue de 69,3 y $24,1 \%$ respectivamente. En el caso de gatos la tenencia de uno o dos animales fue de $62,4 \mathrm{y}$ $22,8 \%$ respectivamente. El promedio de mascotas por vivienda fue de 1,4 para perros y 1,7 para gatos, entre las viviendas que la poseían (tabla 2).

Se estimó una relación persona: perro de 5:1 y persona: gato de 19,5:1. Considerando que la proyección de población humana en el distrito de San Borja para el 2017 fue de 112712 habitantes (Instituto Nacional de Estadística e Informática [INEI], 2017), la población estimada en perros fue de 22543 y en los gatos de 5781 para el año 2017.

El porcentaje de supervivencia sobre 158 crías de perros y 57 crías de gatos nacidas fue estimado en $88,6 \pm 4,9 \%$ y $88,4 \% \pm 9,4 \%$ para perros y gatos respectivamente. Sobre 74 y 12 encuestas contestadas para estimar la esperanza de vida de perros y gatos, esta fue calculada en $9,44 \pm 1,2$ y 7,38 $\pm 3,4$ para las respectivas especies. La causa de muerte mencionada 
Tabla 1. Distribución de la tenencia de perros y gatos según tipo de vivienda. Distrito de San

Borja, Lima - Perú (julio, 2017).

\begin{tabular}{cccccc}
\hline Tipo de vivienda & $\begin{array}{c}\text { Número de } \\
\text { viviendas } \\
\text { encuestadas }\end{array}$ & Nro. & $\%$ & Nro. & $\%$ \\
\hline Casa & 503 & 272 & 54,1 & 63 & 12,5 \\
Departamento & 355 & 190 & 53,5 & 36 & 10,1 \\
Otros & 13 & 5 & 38,5 & 2 & 15,4 \\
Total & 871 & 472 & 54,2 & 101 & 11,6 \\
& & & & & \\
\hline
\end{tabular}

Tabla 2. Promedio de animales de compañía según tipo de vivienda que los poseían. Distrito de San Borja, Lima - Perú (julio, 2017).

\begin{tabular}{lcccccc}
\hline $\begin{array}{c}\text { Tipo de } \\
\text { Vivienda }\end{array}$ & $\begin{array}{c}\text { Nro. Viviendas } \\
\text { encuestadas }\end{array}$ & $\begin{array}{c}\text { Perros } \\
\text { perros }\end{array}$ & $\begin{array}{c}\text { Promedio/ } \\
\text { vivienda }\end{array}$ & $\begin{array}{c}\text { Nro. viviendas } \\
\text { encuestadas }\end{array}$ & $\begin{array}{c}\text { Total de } \\
\text { gatos }\end{array}$ & $\begin{array}{c}\text { Promedio/ } \\
\text { vivienda }\end{array}$ \\
\hline Casa & 277 & 412 & 1,5 & 63 & 113 & 1,8 \\
Departamento & 190 & 245 & 1,3 & 36 & 49 & 1,4 \\
Otros & 5 & 7 & 1,4 & 2 & 8 & 3,5 \\
Total & 472 & 664 & 1,4 & 101 & 170 & 1,7 \\
\hline
\end{tabular}

Tabla 3. Distribución de las causas de muerte de los animales de compañía según opinión de los encuestados. Distrito de San Borja, Lima - Perú (julio, 2017)

\begin{tabular}{lcccc}
\hline \multirow{2}{*}{ Causa de muerte } & \multicolumn{2}{c}{ Perros } & \multicolumn{2}{c}{ Gatos } \\
& Nro. & $\%$ & Nro. & $\%$ \\
\hline Accidente & 20 & 27,0 & 1 & 8,3 \\
Enfermedad & 25 & 33,8 & 9 & 75 \\
Envenenamiento & 1 & 1,4 & - & - \\
Vejez & 28 & 37,8 & 2 & 16,7 \\
\multicolumn{1}{c}{ Total } & 74 & 100 & 12 & 100 \\
\hline
\end{tabular}

Tabla 4. Características demográficas cualitativas de los perros criados en el distrito de San Borja, Lima Perú (julio, 2017)

\begin{tabular}{cccc}
\hline Variable & Estrato & Nro. & $\%$ \\
\hline Sexo & Macho & 369 & 57,2 \\
$(\mathrm{n}=645)$ & Hembra & 276 & 42,8 \\
Raza & Puro & 428 & 67,5 \\
$(\mathrm{~N}=634)$ & Cruzado & 206 & 32,5 \\
& Pequeño & 266 & 45,6 \\
Tamaño & Mediano & 231 & 39,6 \\
$(\mathrm{n}=583)$ & Grande & 86 & 14,8 \\
Esterilización & Si & 190 & 29,6 \\
$(\mathrm{n}=642)$ & No & 452 & 70,4 \\
Utilidad del & Mascota & 631 & 97,9 \\
can & Guardián & 6 & 0,9 \\
$(\mathrm{n}=645)$ & Reproducción & 8 & 1,2 \\
\hline
\end{tabular}

Tabla 5. Características demográficas cualitativas de los gatos domésticos criados en el distrito de San Borja, Lima - Perú (julio, 2017)

\begin{tabular}{cccc}
\hline Variable & Estrato & Nro. & $\%$ \\
\hline Sexo & Macho & 76 & 44,7 \\
$(\mathrm{n}=170)$ & Hembra & 94 & 55,3 \\
Raza & Puro & 33 & 20,4 \\
$(\mathrm{~N}=162)$ & Cruzado & 129 & 79,6 \\
Esterilización & Si & 106 & 63,5 \\
$(\mathrm{n}=167)$ & No & 61 & 36,5 \\
Utilidad del felino & & & \\
$(\mathrm{n}=170)$ & Mascota & 170 & 100,0 \\
\hline
\end{tabular}

Tabla 6. Edad de perros y gatos criados por los encuestados en el distrito de San Borja, Lima - Perú (julio, 2017)

\begin{tabular}{lcc}
\multicolumn{1}{c}{ Variables } & Perros & Gatos \\
\hline Nro. de animales & 645 & 170 \\
Promedio de edad & 4,26 & 2,52 \\
Desviación estándar & 3,48 & 2,35 \\
Valor mínimo & 0,17 & 0,02 \\
Valor máximo & 18 & 16 \\
Mediana & 3 & 2 \\
Moda & 3 & 2 \\
\hline
\end{tabular}


con mayor frecuencia fue vejez o fallecimiento por causas naturales en perros, mientras que en los gatos fueron las enfermedades (tabla 3 ).

El principal uso de los perros fue como animales de compañía (97,9\%). Sobre los indicadores demográficos para esta especie, se encontró una mayor tendencia a tener animales machos $(57,2 \%)$, de raza pura $(67,5 \%)$, no esterilizados $(70,4 \%)$ y de tamaño pequeño $(45,6 \%)$ (tabla 4). En el caso de los gatos, el total de encuestados mencionó que la utilidad era la de mascota (100\%). Del mismo modo, se presentó una mayor predominancia de gatos hembras $(55,3 \%)$, esterilizados $(63,5 \%)$ y de razas mestizas o cruzados $(79,6 \%)$ (tabla 5). En la tabla 6 se presenta la edad promedio de las mascotas que en el momento de la encuesta se encontraban en el distrito.

\section{DISCUSIÓN}

El estudio de demografía de animales domésticos realizado en San Borja complementa la información existente a la fecha respecto a las características de la población de perros y gatos en el Perú y se espera contribuya a la adecuada planificación de los programas de vacunación para el control de la Rabia y los programas de Tenencia Responsable de Animales de Compañía que la Municipalidad de San Borja proponga. En el primer caso, la información es relevante debido a que según la OMS, para alcanzar un adecuado control, prevención, y de ser el caso, erradicación de la rabia en perros, los programas deben incluir campañas anuales que alcancen una cobertura de vacunación de por lo menos $70 \%$ de la población (World Health Organization [WHO], 2013). En el caso del Perú, según la "Norma técnica de salud para la prevención y control de rabia humana en el Perú", se sugiere que la cobertura debe de alcanzar no menos del $80 \%$ de la población estimada (MINSA, 2017).

La tenencia de animales de compañía es mayoritaria en las viviendas del distrito, siendo superior la posesión de perros sobre los gatos. Los beneficios tanto en la salud física como mental de sus propietarios estaría entre las razones que avalan su tenencia (Hugues et al., 2013; Cuch, 2004; Gómez et al., 2007), siendo el perro la especie que se vincula de manera más estrecha con las personas. Respecto al número de animales de compañía por vivienda, se observa que la mayoría posee un perro o un gato, lo que podría estar asociado a la disponibilidad de espacio, así como a la reglamentación establecida por la Municipalidad de San Borja, que limita el número de animales de compañía que puede mantenerse en una vivienda a solo dos: Ordenanzas $\mathrm{N}^{\circ} 484$ y 581 MSB (Municipalidad Distrital de San Borja, 2012; Municipalidad Distrital de San Borja, 2017).

En un estudio similar realizado en VentanillaCallao (Rendón et al., 2018), también encontró un mayor porcentaje de perros sobre gatos en los hogares encuestados ( $62 \%$ y $40 \%$ respectivamente). En el caso de perros, Arauco et al. (2014) encontraron que el 58,2\% de las viviendas de San Martin de Porres los poseían y Soriano et al. (2017) reportó valores del 60,5\% en Comas, ambos distritos de Lima - Perú. El mismo comportamiento se observó en estudios realizados en el interior del país. En Huaraz - Ancash el 77,5\% de las viviendas poseían perros (Morales, 2014) y en Tarapoto esta proporción alcanzó al $60 \%$ (Chu, 2016). En otros países también predominó la tenencia de perros sobre gatos como en Chile donde un estudio encontró que el 53,3\% de viviendas poseían perros y solo el 17,8\% tenían gatos (Rojas, 2005).

El promedio de perros por vivienda hallado es similar al encontrado en los estudios realizados en Ventanilla (Rendón et al., 2018), San Martin de Porres (Arauco et al., 2014) y Comas (Soriano et al., 2017) en donde se encontraron promedios de 1,8, 1,6 y 1,7 perros por vivienda, respectivamente. Sin embargo el valor reportado fue mayor a los obtenidos en estudios realizados en Quito (Vinueza, 2015), Argentina (Brusoni, Dezzotti, Canigia, y Lara, 2007) y la Comuna de Lo Prado en Chile (Rojas, 2005) donde se hallaron promedios de $0,86,0,62$ y 0,77 perros por vivienda, respectivamente. El promedio de gatos por vivienda fue similar al resultado encontrado en Ventanilla en donde se reportó un promedio de gatos por vivienda de 1,8 gatos (Rendón et al., 2018); pero mayor al hallado en la Comuna de Santiago (Bustamante, 2008) y la comuna de Lo Prado en Chile (Rojas, 2005), en donde se reportaron promedios de 0,46 y 0,27 gatos por vivienda, respectivamente.

Esto se debería a la forma como se están expresando los resultados. Mientras que en los estudios del Perú se considera el promedio de animales de compañía como el cociente del total de animales entre el total de viviendas que lo poseen, los estudios internacionales consideran el promedio al cociente del total de animales/ entre el total de viviendas encuestadas. La ventaja de los primeros resultados seria el tener una idea más cercana a la cantidad de animales de compañía que se esperaría encontrar en las viviendas que lo poseen. 
La relación persona: perro reportada en el estudio fue de 5:1, valor cercano a lo reportado en otros distritos de Lima. En Ventanilla esta relación fue de 4:1 (Rendón et al., 2018), en Comas 5,74: 1 (Soriano et al., 2017) y en la comunidad de Jardines de Manchay en Pachacamac fue de 3,9:1 (Málaga et al., 2014). Esto contrasta con los resultados obtenidos en San Martin de Porres donde dicha relación fue de 7:1 (Arauco et al., 2014). Se requiere un análisis mayor cada vez que aparentemente las condiciones sociales, culturales y económicas de los distritos no presenten una tendencia con la relación persona: perro. En otros países las relaciones también son variadas. En Colombia esta relación fue de 12,8:1 (Santa Fe, 2004), en contraste a lo observado en Quito, donde se obtuvo una relación persona: perro de 2,23:1 (Vinueza, 2015). La relación persona: gato encontrada en el estudio $(19,5: 1)$ difiere a lo observado en Ventanilla, donde esta relación fue de 5,34:1, lo que se atribuyó al uso de estos animales como controladores biológicos de roedores (Rendón et al., 2018). Los hallazgos en gatos es menor dentro de la literatura, encontrándose que el reportado en la Ciudad de Torreón en México (19:1) (Bautista, 2013) es similar al encontrado en San Borja.

La esperanza de vida promedio encontrada para los perros en el estudio fue mayor a la de Ventanilla (Rendón et al., 2018) y San Martin de Porres (Arauco et al., 2014) donde se alcanzó promedios de 4,21 y 5,2 años respectivamente. Así mismo, el resultado en gatos también fue superior al hallado en Ventanilla donde los gatos sobrevivían en promedio 2,64 años (Rendón et al., 2018). Estos resultados podrían deberse a un mayor cuidado que tienen los dueños por la salud de sus animales de compañía gracias a las características socioeconómicas del distrito en estudio (INEI, 2014).

La principal causa de muerte en perros fue la vejez, lo que difiere del fallecimiento por causas desconocidas y accidentes encontrados en estudios realizados en Chile (Bustamante, 2008; Illanes, 2009) y muerte por aplastamiento para el caso de cachorros reportados en Villa del Mar (Morales, Varas, y Ibarra, 2009). En caso de los gatos, la mayoría de los decesos se debe a enfermedades, lo que podría inferir de que existe una mayor preocupación por la salud de los perros que por la de los gatos por parte de sus dueños o que la detección de la condición de enfermo podría ser más evidente en los perros por sus comportamiento en relación a las personas. En contraste, estudios reportan que en Chile predominó la vejez como principal causa de fallecimiento de los gatos (Bustamante, 2008; Rojas, 2005).
La tasa de supervivencia de crías encontrada en este estudio fue similar para ambas especies (alrededor del $88 \%$ ). Este indicador es importante porque se encuentra relacionado con el crecimiento poblacional de animales de compañía. La tasa de supervivencia en perros fue mayor a las encontradas en otros estudios. En Ventanilla fue de 56,2\% (Rendón et al., 2018), San Martin de Porres de 75,7\% (Arauco et al., 2014), Comas de $64,7 \%$ (Soriano et al., 2017) y Huaraz- Ancash de $70 \%$ (Morales, 2014). Sin embargo, fue menor a las encontradas en estudios similares en Chile, donde se obtuvieron tasas del 90,8\% (Bustamante, 2008) y 90,6\% (Rojas, 2005). La comparación con otras realidades peruanas indicaría que la condición social, económica y cultural de los habitantes del distrito de San Borja favorecería esta mayor supervivencia. Para el caso de gatos, el resultado también fue superior al encontrado en Ventanilla donde se reportó una supervivencia de $66,4 \%$ (Rendón et al., 2018), pero se hallaron resultados más cercanos en estudios realizados en Chile, donde la supervivencia alcanzo $86,3 \%$ (Bustamante, 2008) y 72,8\% (Rojas, 2005).

Las edades promedio para ambas especies, indican una predominancia de animales en edad adulta. El resultado en perros fue mayor al encontrado en San Martin de Porres y Comas, donde predominó una población más joven con un promedio de 2,7 años y 3 años respectivamente (Arauco et al., 2014; Soriano et al., 2017). Por otro lado, se encontró resultados similares en estudios realizados en Argentina, donde se obtuvo un promedio de 4,9 años (Brusoni et al., 2007) y en Quito con perros de edades entre 1 y 5 años (Vinueza, 2015). Así mismo, la edad promedio de gatos (2,52 años) también fue superior a las encontradas en estudios similares realizados en Chile, con un promedio de edad de 1 a 1,9 años (Gallardo, 2003), en Colombia con promedios de 1 a 2 años (Santa Fe, 2004) y Bolivia con promedios de 4 meses a 1 año (Loza, 2014).

En cuanto a las características demográficas en perros, se encontró un mayor porcentaje de machos en relación con las hembras, similar tendencia que los estudios en Ventanilla donde alcanzó el 59,2\% (Rendó et al., 2018), San Martin de Porres con 56,6\% (Arauco et al., 2014) y Huaraz -Ancash con 61\% (Morales, 2014), demostrando así una preferencia de perros de este sexo debido posiblemente a su mayor facilidad de crianza en comparación a la de las hembras, las cuales suelen causar problemas particularmente durante el periodo de celo (Güttler, 2005; Vinueza 2015). 
En contraste con otros estudios realizados en el país (Rendón et al., 2018; Arauco et al., 2014; Morales, 2014; Soriano et al., 2017) y en otros países de Latinoamérica (Vinueza, 2015; Gallardo, 2003; Santa Fe, 2004) donde predominó principalmente la posesión de animales de raza mestiza, en el estudio se encontró un mayor porcentaje de perros de raza pura. Esto podría deberse a que, por las características socioeconómicas de San Borja (INEI, 2014), la población tendría una mayor disponibilidad de recursos económicos para la adquisición de perros de esta clase. Además, se encontró una mayor predilección por la tenencia de perros de razas pequeñas quizás por las mayores ventajas en términos de espacio que conlleva la crianza de este tipo de perros en comparación a los de mayor tamaño, lo que se haría evidente especialmente en el caso de departamentos. El resultado fue diferente al encontrado en San Martin de Porres (Arauco et al., 2014), Ventanilla (Rendón et al., 2018) y en Chile (Gallardo, 2003), donde predominaron principalmente los perros de razas medianas y grandes, lo que podría estar relacionado al uso de los mismos para guardianía y seguridad (Bahamonde, 2010).

A diferencia de los perros, los gatos hembra predominaron en el distrito, lo cual fue semejante a los resultados encontrados en Bolivia (Loza, 2014) y Santiago de Chile (Bustamante, 2008); pero difirió a los hallados en Colombia (Santa Fe, 2004) y Quito (Vinueza, 2015), donde predominaron principalmente los machos de esta especie. No encontrándose razones que justifiquen este hallazgo en la literatura revisada. Sin embargo podría estar relacionado a las características fisiológicas y etológicas que muestran las gatas durante el celo. Estas se describen con un comportamiento afectuoso, menos hostil con el macho y presencia de fuertes vocalizaciones (Giménez et al., 2006). Razón por la cual muchos propietarios sienten la necesidad de esterilizar a sus animales de compañía (Rojas, 2017).

La tenencia de gatos de raza mestiza o cruzada predominó en el distrito, pudiéndose deber a una menor oferta de animales de raza y mayor disponibilidad de gatos sin linaje por medio de campañas de adopción, así como por el desconocimiento sobre las diversas razas de gatos existentes (Vinueza, 2015). Hallazgos semejantes fueron encontrados en estudios en Colombia (Santa Fe, 2004), Bolivia (Loza, 2014). Quito (Vinueza, 2015) y la Comuna de Santiago en Chile (Bustamante, 2008).
Finalmente, la principal razón de la tenencia tanto de perros como de gatos en el distrito fue para ser mascotas o animales de compañía. En contraste un estudio en la comuna de Calera de Tango en Chile, encontró un predominio de la función de guardián para los perros, lo cual se debe posiblemente al predominio de la población rural en dicha comuna y por lo tanto se necesita que los perros brinden protección contra robos a las viviendas (Illanes, 2009).

Los datos ofrecidos por los estudios demográficos en animales de compañía deben de ser considerados como indicadores basales que permitan evaluar el impacto de las intervenciones en salud pública, entre ellas las de campañas de vacunación antirrábica y tenencia responsable de animales de compañía en el distrito de San Borja.

\section{CONCLUSIONES}

El estudio de indicadores demográficos de la población de perros y gatos con dueño en el distrito de San Borja, Lima - Perú, 2017, llega a las siguientes conclusiones:

El $54,2 \%$ y $11,6 \%$ de las viviendas encuestadas tenían perros y gatos respectivamente.

El promedio de animales por vivienda fue de 1,4 para perros y 1,7 para gatos, entre las viviendas que poseían los respectivos animales de compañía.

Se estimó una relación persona:perro de 5:1 y persona:gato de 19,5:1.

La población estimada de perros fue de 22543 y de gatos de 5781 para el año 2017.

El porcentaje de supervivencia de las crías estimado fue de 88,6 y $88,4 \%$ y la esperanza de vida estimada fue de 9,44 y 7,38 años, para perros y gatos respectivamente.

En caso de perros el principal uso fue como mascota (97,9\%), predominando la tenencia de machos $(57,2 \%)$, de raza pura $(67,5 \%)$, no esterilizados $(70,4 \%)$, tamaño pequeño $(45,6 \%)$ y con una edad promedio de 4,26 años.

En caso de los gatos el único uso fue como mascota (100\%), predominaron las hembras $(55,3 \%)$, esterilizadas $(63,5 \%)$, raza cruzada $(79,6 \%)$ y edad promedio de 2,52 años. 


\section{Correspondencia}

Raquel Arellano Bastidas

Correo electrónico: rarba9107@gmail.com

\section{REFERENCIAS BIBLIOGRAFICAS}

1. Arauco, D., Falcón, N., León, D., \& Urbina, B. (2014). Indicadores demográficos y estimación de la población de canes con dueño en el distrito de San Martin de Porres. Rev Salud Tecno Vet, 2, 83-92.

2. Bahamonde, R. (2010). Muestreo censal canino en la Ciudad de Puerto Natales. (Tesis para Título de Ingeniero en Ejecución de Recursos Naturales, Universidad de Magallanes, Puerto Natales, Chile).

3. Bautista, J. (2013). Estudio demográfico de gatos con dueño en la ciudad de Torreón, Coahuila, México. (Título de Médico Veterinario Zootecnista, Universidad Antonio Narro, Saltillo, México)

4. Botero, T. (2009). Tenencia responsable de mascotas. Rev Col de Cienc Animal, 2 (2), 9-12.

5. Brusoni, C., Dezzotti, A., Canigia, J., \& Lara, J. (2007). Tamaño y estructura de la población canina en San Martin de los Andes (Neuquén). Analecta Veterinaria, Argentina, 27(1), 11-23.

6. Bustamante, S. (2008). Demografia en las poblaciones de canes y felinos en la comuna de Santiago. (Título de Médico Veterinario, Universidad de Chile, Santiago, Chile).

7. Chu, A. (2016). Diagnóstico sobre tenencia responsable de animales de compañia en trece sectores del distrito de Tarapoto, provincia de San Martin. (Tesis para Título de Médico Veterinario. Universidad Nacional de San Martin, Tarapoto, Perú).

8. Congreso de la República del Perú. (14 de diciembre de 2001). Ley $N^{\circ}$ 27596. Ley que regula el régimen jurídico de canes. (pp. 213876-213878). Lima, Perú: Diario Oficial El Peruano. Recuperado de: http://busquedas.elperuano.com.pe/download/ full/7SXeVAz2Ket8qUh jMAog-

9. Cuch, L. (2004). Animales de compañia beneficios $e$ inconvenientes. LOS MONOGRÁFICOS DE B.MM, 6(1), 1-5. Recuperado de: https://www.diba. cat/c/document_library/get_file?uuid=0ad3534fc7ee-4bb6-925f-e 98f9d1160e 3\&groupId=7294824

10. Gallardo, M. (2003). Características demográficas de la población canina y recuento de la población felina en la ciudad de La Unión. (Tesis para Título Profesional de Médico Veterinario, Universidad Austral de Chile, Valdivia, Chile).

11. Giménez, F., Stornelli, M., Savignone, C., Tittarelli, C., De la Sota, R., \& Stornelli, M. (2006). Fisiología reproductiva y control de los ciclos estrales en la gata doméstica. Analecta Veterinaria, 26, 38-43.

12. Gómez, L., Atehortua, C., \& Orozco, S. (2007). La influencia de las mascotas en la vida humana. Rev Col Cienc, 20 (3), 377-386

13. Güttler, V. (2005). Análisis de algunas características de la población canina relacionadas con mordeduras e hidatidosis humana en la provincia de Valdivia. (Tesis pata Título de Médico Veterinario, Universidad Austral de Chile, Valdivia, Chile).

14. Hugues, B., Álvarez, A., Ledón, L., Mendoza, M., Castelo, L., \& Domínguez, E. (2013). Efectos beneficiosos de los animales de compañía para los pacientes con enfermedades cardiovasculares. CorSalud, 5(2), 226-229.

15. Illanes, J. (2009). Demografia en las poblaciones de perros y gatos en el Área Rural y Urbana de la Comuna de la Calera del Tango. (Título de Médico Veterinario, Universidad de Chile, Maipo, Santiago).

16. Instituto Nacional de Estadística e Informática. (2014). Una mirada a Lima Metropolitana. Lima, Perú: Instituto Nacional de Estadística e Informática. Recuperado de: https://www.inei.gob.pe/media/ MenuRecursivo/publicaciones_digitales/Est/ Lib1168/libro.pdf

17. Instituto Nacional de Estadística e Informática. (2017). Provincia de Lima: Compendio estadístico 2017. Lima, Perú: Instituto Nacional de Estadística e Informática. Recuperado de: https://www.inei.gob. pe/media/MenuRecursivo/publicaciones_digitales/ Est/Lib1477/libro.pdf

18. León, D., Soriano, J., Arauco, D., \& Falcón, N. (2014). Estimación de la población de canes con dueño y canes vagabundos: Importancia para la Salud Pública. MV Revista de Ciencias Veterinarias Perú, 30 (3), 1-4. Recuperadode: https://favez.cayetano.edu.pe/images/ ARTICULOS_TECNICOS/ESTIMACION_DE_ LA_POBLACION_DE_PERROS_CON DUENO_Y_PERROS_VAGABUNDOS IMPORTANCIAIA_PARA_LA_SALUD_PUBLICA. pdf

19. Loza, A. (2014). Caracterización de la población canina y felina en Santa Cruz de la Sierra. Santa Cruz de la Sierra: Universidad Autónoma Gabriel Rene Moreno.

20. Málaga, H. (1973). Características de la población canina y felina en Lima Metropolitana. (Tesis para optar el título de Médico Veterinario, Universidad Nacional Mayor de San Marcos, Lima, Perú).

21. Málaga, H., Piña, J., Denegri, A., Stefan, T., Cedamanos, D., Alvarado, D.,...Taipe, C. (2014). Priorización de problemas de salud comunitaria en una localidad de Lima Metropolitana en el marco del Programa de Municipalidades Saludables. Revista Peruana de Epidemiología, 18(2), 1-4.

22. Ministerio de Salud (2017). NTS N ${ }^{a} 131-M I N S A / 2017 /$ DGIESP. Norma Técnica de Salud para la Prevención y Control de la Rabia Humana en el Perú. Lima, Perú: Ministerio de Salud. 
23. Morales, A., Varas, C., \& Ibarra, L. (2009). Caracterización demográfica de la población de perros de Viña del Mar. Revista de Chile, 41, 89-95.

24. Morales, D. (2014). Estimación de la población de canes con dueño en la Ciudad de Huaraz, departamento de Ancash - Perú. (Tesis para optar el título de Médico Veterinario Zootecnista. Universidad Peruana Cayetano Heredia, Lima, Perú).

25. Municipalidad Distrital de San Borja (2012). Ordenanza $N^{\circ}$ 484-MSB/2012. Ordenanza que norma el Régimen de Tenencia Responsable y registro de canes y felinos en el Distrito de San Borja. Lima, Perú: Consejo de la Municipalidad Distrital de San Borja. Recuperado de: http://www.munisanborja.gob. pe/index.php/revista-san-borja/cat_view/21normas/14-ordenanzas-municipales/280ordenanzas-2012-2013.html? limit $=5 \&$ order $=$ hits \&d $i r=D E S C$

26. Municipalidad Distrital de San Borja (2017). Ordenanza $N^{\circ}$ 581-MSB/2017. Ordenanza que establece el Régimen de animales domésticos en el Distrito de San Borja. Lima, Perú: Consejo de la Municipalidad Distrital de San Borja. Recuperado de: https://busquedas.elperuano.pe/download/url/ establecen-regimen-juridico-de-tenencia-deanimales-domestic-ordenanza-no-58 msb-1515705-1

27. Rendón, D., Quintana, E., Door, I., Vicuña, F., León, D., \& Falcón, N. (2018). Parámetros demográficos en la población de canes y gatos domésticos en asentamientos humanos del distrito de Ventanilla, Callao-Perú. Rev Inv Vet Perú, 29(1), 217-225.
28. Rojas, A. (2005). Demografía en las poblaciones de canes y felinos en la Comuna de Lo Prado. (Título de Médico Veterinario, Universidad de Chile, Santiago, Chile).

29. Rojas, P. (2017). Características de los perros y gatos bajo control reproductivo quirúrgico registrados en la Municipalidad de Los Olivos: Periodo 2015-2016. (Título de Médico Veterinario, Universidad Peruana Cayetano Heredia, Lima, Perú).

30. Santa Fe, L. (2004). Muestreo para determinar la población de caninos y felinos en el Municipio de Santiago de Cali. Cali, Colombia: Secretaria de Salud Pública Municipal de Santiago de Cali-Centro de Zoonosis. Recuperado de: http://www.cali.gov.co/ publico2/documentos/varios/tenenciamascotas.pdf

31. Soriano, J., Nuñez, J., León, D., \& Falcón, N. (2017). Estimación de la población de canes con dueño en el distrito de Comas, Lima-Perú. MV Rev de Cien Vet, $33(2), 5-10$.

32. Venegas, J. (2014). Actualización y comparación de situación demográfica en perros y gatos en la Comuna de lo Prado (Año 2004-Año 2013). (Tesis para Título Profesional de Médico Veterinario, Universidad de Chile, Santiago, Chile).

33. Vinueza, N. (2015). Determinación de la población de mascotas en hogares de la Parroquia de San Bartolo del Distrito Metropolitano de Quito. (Tesis para Título de Médico Veterinario Zootecnista, Universidad de Las Américas, Quito, Ecuador).

34. World Health Organization (2013). WHO Expert Committee on rabies: second report. Geneva: World Health Organization. Recuperado de: https://apps. who.int/iris/handle/10665/85346 